\title{
REMARKS ON THE THIN OBSTACLE PROBLEM AND CONSTRAINED GINIBRE ENSEMBLES
}

\author{
ARAM L. KARAKHANYAN
}

\begin{abstract}
We consider the problem of constrained Ginibre ensemble with prescribed portion of eigenvalues on a given curve $\Gamma \subset \mathbb{R}^{2}$ and relate it to a thin obstacle problem. The key step in the proof is the $H^{1}$ estimate for the logarithmic potential of the equilibrium measure. The coincidence set has two components: one in $\Gamma$ and another one in $\mathbb{R}^{2} \backslash \Gamma$ which are well separated. Our main result here asserts that this obstacle problem is well posed in $H^{1}\left(\mathbb{R}^{2}\right)$ which improves previous results in $H_{\text {loc }}^{1}\left(\mathbb{R}^{2}\right)$.
\end{abstract}

\section{INTRODUCTION}

Let $\Gamma$ be a regular curve in $\mathbb{R}^{2}$ with locally finite length and $\mathcal{M}_{a}$ the set of all probability measures such that

$$
\mu(\Gamma) \geq a, \quad a \in(0,1)
$$

By an abuse of notation we let $\Gamma: \mathbb{R} \rightarrow \mathbb{R}^{2}$ be the arc-length parametrization of the curve such that

$$
|\dot{\Gamma}(t)|=1, \quad t \in \mathbb{R} .
$$

In this paper we consider the minimizers of the energy

$$
I[\mu]=\iint \log \frac{1}{|x-y|} d \mu(x) d \mu(y)+\int Q d \mu
$$

where $Q(x)$ is a given function such that the weight function $w=e^{-Q}$ on $\mathbb{R}^{2}$ is admissible (see Definition 1.1 p.26 [8]). This means that $w$ satisfies the following three conditions:

(H1) $w$ is upper semi-continuous;

(H2) $\left\{w \in \mathbb{R}^{2}\right.$ s.t. $\left.w(z)>0\right\}$ has positive capacity;

(H3) $|z| w(z) \rightarrow 0$ as $|z| \rightarrow \infty$.

In higher dimensions $\mathbb{R}^{d}, d \geq 3$ one can consider more general kernels

$$
K(x-y)= \begin{cases}\log \frac{1}{|x-y|}, & d=2, \\ \frac{1}{|x-y|^{d-2}}, & d \geq 3,\end{cases}
$$

with $\Gamma$ being a Lyapunov surface in $\mathbb{R}^{d}$ and define the energy as follows

$$
I[\mu]=\iint K(x-y) d \mu(x) d \mu(y)+\int Q d \mu .
$$

2000 Mathematics Subject Classification. Primary 35R35, 31A35, 49K10, 60B20.

Keywords: Obstacle problem, thin obstacle, free boundary, global regularity. 
In this note we mostly confine ourselves with quadratic potentials $Q(x)=|x|^{2}$ in $\mathbb{R}^{2}$, although all our results remain valid for more general $Q$ satisfying $(\mathbf{H 1})-(\mathbf{H} 3)$. Furthermore, our main result on global $L^{2}$ estimate of the gradient of the equilibrium potential with kernel $K(x-y)=|x-y|^{-d}$ remains valid in in $\mathbb{R}^{d}, d \geq 3$, see Theorem 4.1 .

The functional $I[\mu]$, with $Q=|x|^{2}, d=2$, arises in the description of the convergence of the spectral measure of square $N \times N$ matrices with complex independent, standard Gaussian entries (i.e., the Ginibre ensemble) as $N \rightarrow \infty$. In case when there are no constraints imposed on the eigenvalues, it is well known that the eigenvalues spread evenly in the ball of radius $\sqrt{N}$, and after renormalization by a factor $\frac{1}{\sqrt{N}}$ the normalized spectral measure converges to the characteristic function of the unit disc. This is known as the circular law [4], [2]. In this context the functional $I$ is used to prove large deviation principles for the spectral measure.

If one demands that the eigenvalues are real (i.e. when $a=1, \Gamma=\mathbb{R}$ ) we get the so called semicircle law. More generally, one can demand that a portion of eigenvalues is contained in a prescribed set $\Gamma$. This is considered in [2] when a portion of eigenvalues are contained in an open bounded subset of $\mathbb{R}^{2}$ and in [4] when $\Gamma$ is a line. These problems can be related to the thin obstacle and obstacle problems respectively. The key step in proving this is to establish $H_{l o c}^{1}\left(\mathbb{R}^{2}\right)$ estimates for the logarithmic potential

$$
U^{\mu_{a}}=K * \mu_{a}
$$

of the corresponding equilibrium measure. The aim of this note is to show that the thin obstacle problem is well-posed in $H^{1}\left(\mathbb{R}^{2}\right)$ by showing that in fact $U^{\mu_{a}} \in H^{1}\left(\mathbb{R}^{2}\right)$, see Theorem 4.1. This improves the previous results in [2] and [4].

The paper is organized as follows: In the next section we prove the existence and uniqueness of the equilibrium measure $\mu_{a}$ minimizing the energy $I[\mu]$. In section 3 we discuss some basic properties of $\mu_{a}$. In particular we show that there are two positive constants $A_{\Gamma}$ and $A_{0}$ such that $2 U^{\mu_{a}}+Q=A_{\Gamma}$ on $\operatorname{supp} \mu_{a} \cap \Gamma$ and $2 U^{\mu_{a}}+Q=A_{0}$ on supp $\mu_{a} \backslash \Gamma$. Furthermore, $A_{\Gamma}>A_{0}$. This fact will be used later to show that $\operatorname{supp} \mu_{a} \backslash \Gamma$ and $\operatorname{supp} \mu_{a} \cap \Gamma$ are disjoint.

Our main result Theorem 4.1 is contained in section 4. To prove it we study the Fourier transformations of $U^{\mu_{a}}$ and $\mu_{a}$. It leads to some integral identity involving Bessel functions. This approach is based on a method of L. Carleson [3]. Finally, combining the results obtained, in section 5 we show that $U^{\mu_{a}}$ solves the obstacle problem where the obstacle is given by

$$
\psi(x)= \begin{cases}\frac{1}{2}\left(A_{\Gamma}-|x|^{2}\right) & \text { if } x \in \Gamma, \\ \frac{1}{2}\left(A_{0}-|x|^{2}\right) & \text { if } x \in \mathbb{R}^{2} \backslash \Gamma .\end{cases}
$$

\section{EXISTENCE OF MINIMIZERS}

In this section we show the existence of a unique equilibrium measure.

Theorem 2.1. Suppose $d=2, \Gamma \subset \mathbb{R}^{2}$ is a regular $C^{1, \alpha}$ smooth planar curve without self-intersections. There is a unique minimizer $\mu_{a} \in \mathcal{M}_{a}$ of $I[\mu]$ such that

$$
I\left[\mu_{a}\right]=\inf _{\mu \in \mathcal{M}_{a}} I[\mu] .
$$

Proof. Observe that the uniqueness follows from the convexity of $\mathcal{M}_{a}$ and can be proved as in [4]. Moreover, $I[\mu]$ is also semicontinuous. Thus, we have to show that $I[\mu]$ is bounded by below for all $\mu \in \mathcal{M}_{a}$ 
and there is at least one $\mu_{0}$ such that $I[\mu]$ is finite. The lower bound follows as in the proof of Theorem 1.3 (a) p. $27[8]$.

It remains show that the $\inf _{\mu \in \mathcal{M}_{a}} I[\mu]<\infty$. Let $\chi_{D}$ denote the characteristic function of the set $D$ and take

$$
\mu=a \frac{1}{L} \mathcal{H}^{1}\left\llcorner(\Gamma \cap \Omega)+(1-a) \frac{1}{|B|} \chi_{B}\right.
$$

where $B=B_{\rho}(z)=\left\{x \in \mathbb{R}^{2}:|x-z|<\rho\right\}$ with small $\rho$ such that $B \subset \Omega, \Omega \subset \mathbb{R}^{2}$ is a compact, $L=\mathcal{H}^{1}(\Gamma \cap \Omega)>0$, and $\operatorname{dist}(\Gamma, B)>0$. Observe that for this choice of $\mu$ we have

$$
\int_{\Omega} \log \frac{1}{|x-y|} d \mu(x)=\frac{1}{L} \int_{0}^{L} \log \frac{1}{|\Gamma(t)-y|} d t+\frac{1}{|B|} \int_{B} \log \frac{1}{|x-y|} d \mu(x) .
$$

Assuming that $\Gamma$ is given by arc-length parametrization we have for the logarithmic energy

$$
\mathcal{L}[\mu]=\frac{a^{2}}{L^{2}} \int_{0}^{L} \int_{0}^{L} \log \frac{1}{|\Gamma(t)-\Gamma(s)|} d t d s+\frac{2 a(1-a)}{L|B|} \int_{0}^{L} \int_{B} \log \frac{1}{|\Gamma(t)-y|} d t d y+\frac{(1-a)^{2}}{|B|^{2}} \int_{B} \int_{B} \log \frac{1}{|x-y|} d x d y .
$$

Since $\operatorname{dist}(\Gamma, B)>0$ then the second integral is bounded. As for the last integral then after change of variables $x-y=\xi$ we have

$$
\int_{B \rho(z)} \log \frac{1}{|x-y|} d x=\int_{B_{\rho}(z-y)} \log \frac{1}{|\xi|} d \xi \leq \int_{B_{2 \rho}(0)} \log \frac{1}{|\xi|} d x<\infty
$$

where we used $|z-y| \leq \rho$ and the fact that $\rho$ is small by construction.

It remains to check that the first integral is finite. Let us fix $s \in[0, L]$ Then we have that

$$
\begin{aligned}
\int_{0}^{L} \log \frac{1}{|\Gamma(t)-\Gamma(s)|} d t & =\int_{-s}^{L-s} \log \frac{1}{|\Gamma(\tau+s)-\Gamma(s)|} d \tau= \\
& =\left.\tau \log \frac{1}{|\Gamma(\tau+s)-\Gamma(s)|}\right|_{-s} ^{L-s}-\int_{-s}^{L-s} \tau \frac{\dot{\Gamma}(\tau+s) \cdot(\Gamma(\tau+s)-\Gamma(s))}{|\Gamma(\tau+s)-\Gamma(s)|^{2}} d \tau= \\
& =(L-s) \log \frac{1}{|\Gamma(L)-\Gamma(s)|}+s \log \frac{1}{|\Gamma(0)-\Gamma(s)|}-I_{0}
\end{aligned}
$$

where $I_{0}$ is the last integral. Using the crude estimate

$$
\begin{aligned}
\left|I_{0}\right| & \leq \int_{-s}^{L-s}|\tau| \frac{|\dot{\Gamma}(\tau+s)|}{|\Gamma(\tau+s)-\Gamma(s)|} d \tau=\int_{-s}^{L-s} \frac{|\tau|}{|\Gamma(\tau+s)-\Gamma(s)|} d \tau= \\
& =\int_{[-s, L-s] \backslash(-\delta, \delta)} \frac{|\tau|}{|\Gamma(\tau+s)-\Gamma(s)|} d \tau+\int_{-\delta}^{\delta} \frac{|\tau|}{|\Gamma(\tau+s)-\Gamma(s)|} d \tau \\
& \leq \frac{4 L^{2}}{C_{\delta}}+\int_{-\delta}^{\delta} \frac{|\tau|}{|\Gamma(\tau+s)-\Gamma(s)|} d \tau
\end{aligned}
$$

because $|\Gamma(\tau+s)-\Gamma(s)| \geq C_{\delta}$ if $|\tau| \geq \delta$. Finally, from $C^{1, \alpha}$ regularity of $\Gamma$ we get

$$
\begin{aligned}
|\Gamma(\tau+s)-\Gamma(s)| & =|\tau|\left|\int_{0}^{1} \dot{\Gamma}(\sigma \tau+s) d \sigma\right| \geq \\
& \geq|\tau|\left(|\dot{\Gamma}(s)|-\int_{0}^{1}|\dot{\Gamma}(\sigma \tau+s)-\dot{\Gamma}(s)| d \sigma\right) \\
& \geq|\tau|\left(1-\delta^{\alpha}\right) .
\end{aligned}
$$


Combining (2.3) with (2.2) we get

$$
\left|I_{0}\right| \leq \frac{4 L^{2}}{C_{\delta}}+2 \delta\left(1-\delta^{\alpha}\right)<\infty
$$

Returning to the first integral in (2.1) we infer

$$
\begin{aligned}
\int_{0}^{L} \int_{0}^{L} \log \frac{1}{|\Gamma(t)-\Gamma(s)|} d t d s \leq & \int_{0}^{L}\left\{(L-s) \log \frac{1}{|\Gamma(L)-\Gamma(s)|}+s \log \frac{1}{|\Gamma(0)-\Gamma(s)|}+\frac{4 L^{2}}{C_{\delta}}+2 \delta\left(1-\delta^{\alpha}\right)\right\} d s \\
\leq & L\left[\frac{4 L^{2}}{C_{\delta}}+2 \delta\left(1-\delta^{\alpha}\right)\right]+L \log \frac{1}{C_{\delta}}+ \\
& +\int_{\delta}^{L-\delta}\left\{(L-s) \log \frac{1}{|\Gamma(L)-\Gamma(s)|}+s \log \frac{1}{|\Gamma(0)-\Gamma(s)|}\right\} d s \\
\leq & C(\delta, L)
\end{aligned}
$$

if we choose $\delta>0$ suitably small. This finishes the proof for $d=2$.

Remark 2.2. If $d \geq 3, Q(x)=|x|^{2}$ then clearly $I[\mu] \geq 0$. The upper estimate for $I[\mu]$ follows from a similar argument if we assume that $\Gamma$ is a Lyapunov surface and take $\mu=a \frac{1}{L} \mathcal{H}^{d-1}\left\llcorner(\Gamma \cap \Omega)+(1-a) \frac{1}{|B|} \chi_{B}\right.$ with $L=\mathcal{H}^{d-1}(\Gamma \cap \Omega)$ and $\operatorname{dist}(B, \Gamma)>0$. Therefore Theorem 2.1 remains valid for $d \geq 3$.

\section{BASIC PROPERTIES OF MINIMIZERS}

In this section we prove some basic properties of the equilibrium measure. The arguments are along the line of those in [2]. Therefore, we mostly focus on those aspects of the proofs which are new or differ essentially. The results to follow are valid in $\mathbb{R}^{d}, d \geq 2$ unless otherwise stated.

Lemma 3.1. Let $\mu_{a}$ be as in Theorem 2.1. Then $\mu_{a}(\Gamma)=a$.

Proof. If the claim fails then $\mu_{a}(\Gamma)>a$. Fix $\delta \in(0, a)$ and let $\mu_{a-\delta}$ be the minimizer of $I[\cdot]$ over $\mathcal{M}_{a-\delta} \supset \mathcal{M}_{a}$. Form $\mu=(1-\varepsilon) \mu_{a}+\varepsilon \mu_{a-\delta}, \varepsilon \in[0,1]$. Clearly, $\mu \in \mathcal{M}_{a}$ if we choose $\varepsilon \delta$ sufficiently small because

$$
\mu(\Gamma)>a+\left[\mu_{a}(\Gamma)-a\right]-\varepsilon \delta
$$

Consequently, we have from the strict convexity of $I$

$$
\begin{aligned}
I\left[(1-\varepsilon) \mu_{a}+\varepsilon \mu_{a-\delta}\right] & <(1-\varepsilon) I\left[\mu_{a}\right]+\varepsilon I\left[\mu_{a-\delta}\right]=I\left[\mu_{a}\right]+\varepsilon\left(I\left[\mu_{a-\delta}\right]-I\left[\mu_{a}\right]\right) \\
& \leq I\left[\mu_{a}\right]
\end{aligned}
$$

which is in contradiction with the fact that $\mu_{a}$ is a minimizer.

Observe that the Fréchet derivative of $I[\mu]$ is $2 U^{\mu_{a}}+Q$ where

$$
U^{\mu_{a}}(y)=\int K(x-y) d \mu_{a}(x) .
$$


It is convenient to consider variations of the equilibrium measure in terms of affine combinations. More precisely, let $\mu_{\varepsilon}=(1-\varepsilon) \mu_{a}+\varepsilon \nu, \nu \in \mathcal{M}_{a}, \varepsilon \in[0,1]$, then by direct computation we have that

$$
\begin{aligned}
I\left[\mu_{\varepsilon}\right]= & (1-\varepsilon)^{2} \iint K(x-y) d \mu_{a}(x) d \mu_{a}(y) \\
& +2 \varepsilon(1-\varepsilon) \iint K(x-y) d \mu_{a}(x) d \nu(y)+\varepsilon^{2} \iint K(x-y) d \nu(x) d \nu(y) \\
& +(1-\varepsilon) \int Q d \mu_{a}+\varepsilon \int Q d \nu \\
= & I\left[\mu_{a}\right]+\varepsilon\left(2 \iint K(x-y) d \mu_{a}(x) d\left(\nu(y)-\mu_{a}\right)+\int Q d\left(\nu-\mu_{a}\right)\right)+O\left(\varepsilon^{2}\right)= \\
= & I\left[\mu_{a}\right]+\varepsilon \int\left(2 U^{\mu_{a}}+Q\right) d\left(\nu-\mu_{a}\right)+O\left(\varepsilon^{2}\right) .
\end{aligned}
$$

Since $\mu_{a}$ is the minimizer then $I\left[\mu_{a}\right] \leq I[\mu]$, and after sending $\varepsilon \rightarrow 0$ it follows that

$$
\int\left(2 U^{\mu_{a}}+Q\right) d\left(\nu-\mu_{a}\right) \geq 0, \quad \forall \nu \in \mathcal{M}_{a} .
$$

Lemma 3.2. Let $A_{\Gamma}=\frac{1}{a} \int_{\Gamma}\left(2 U^{\mu_{a}}+Q\right) d \mu_{a}$ then quasi everywhere

$$
\begin{aligned}
2 U^{\mu_{a}}+Q & =A_{\Gamma} \quad \text { on } \Gamma \cap \operatorname{supp} \mu_{a} \\
& \geq A_{\Gamma} \quad \text { on } \Gamma
\end{aligned}
$$

Similarly, let us denote $A_{0}=\frac{1}{1-a} \int_{\mathbb{R}^{2} \backslash \Gamma}\left(2 U^{\mu_{a}}+Q\right) d \mu_{a}$ then

$$
\begin{aligned}
& 2 U^{\mu_{a}}+Q=A_{0} \quad \text { on } \quad \operatorname{supp} \mu_{a} \backslash \Gamma \\
& \geq A_{0} \text { on } \\
& \mathbb{R}^{2} \backslash\left(\operatorname{supp} \mu_{a} \backslash \Gamma\right) .
\end{aligned}
$$

Furthermore,

$$
A_{\Gamma}>A_{0}
$$

Proof. We first prove (3.3). Suppose that there is a set capacitable $E$ of positive capacity such that $\Gamma \cap E$ has zero capacity and

$$
2 U^{\mu_{a}}+Q<A_{\Gamma}-\delta \text { q.e. on } E
$$

for some positive $\delta$. Let $\mu_{E}$ be the equilibrium measure of $E$ and form $\nu=\mu_{a}\left\llcorner\left(\mathbb{R}^{2} \backslash \Gamma\right)+a \mu_{E}\right.$. Clearly $\nu \in \mathcal{M}_{a}$. Therefore, in view of (3.1) for the measure $\mu_{\varepsilon}=\varepsilon \mu_{a}+(1-\varepsilon) \nu \in \mathcal{M}_{a}$ we get

$$
\begin{aligned}
I\left[\mu_{\varepsilon}\right] & =I\left[\mu_{a}\right]+\varepsilon\left(2 \iint K(x-y) d \mu_{a}(x) d\left(\nu(y)-\mu_{a}\right)+\int Q d\left(\nu-\mu_{a}\right)\right)+O\left(\varepsilon^{2}\right) \\
& =I\left[\mu_{a}\right]+\varepsilon \int_{\Gamma}\left(2 U^{\mu_{a}}+Q\right) d\left(a \mu_{E}-\mu_{a}\right)+O\left(\varepsilon^{2}\right) \\
& =I\left[\mu_{a}\right]+\varepsilon\left(a \int_{\Gamma}\left(2 U^{\mu_{a}}+Q\right) d \mu_{E}-a A_{\Gamma}\right)+O\left(\varepsilon^{2}\right) \\
& <I\left[\mu_{a}\right]-a \varepsilon \delta+O\left(\varepsilon^{2}\right) \\
& <I\left[\mu_{a}\right]
\end{aligned}
$$


if $\varepsilon$ and $\delta$ are sufficiently small. This will be in contradiction with the fact that $\mu_{a}$ is the minimizer. Thus we have proved that $2 U^{\mu_{a}}+Q \geq A_{\Gamma}$ q.e. on $\Gamma$.

Next we show that on supp $\mu_{a} \cap \Gamma$ we have $2 U^{\mu_{a}}+Q=A_{\Gamma}$ q.e. Indeed, from the definition of $A_{\Gamma}$ it follows

$$
a A_{\Gamma}=\int_{\Gamma}\left(2 U^{\mu_{a}}+Q\right) d \mu_{a} \geq a A_{\Gamma}
$$

where the last inequality follows from the first inequality in (3.3). The proof of (3.4) is similar. In order to prove the last claim $A_{\Gamma}>A_{0}$ we first observe that there exists a measure $\nu \in \mathcal{M}_{a}$ such that

- $a>\nu(\Gamma)$,

- $I[\nu] \leq I\left[\mu_{a}\right]$.

First notice that $\mathcal{M}_{a} \subset \mathcal{M}_{a-\delta}$ for $\delta \in(0, a)$. Fix such $\delta>0$ and let $\mu_{a-\delta}$ be the minimizer of $I[\cdot]$ over $\mathcal{M}_{a-\delta}$. Then by Lemma $3.1 \mu_{a-\delta}(\Gamma)=a-\delta<a$ and $I\left[\mu_{a-\delta}\right]=\inf _{\mathcal{M}_{a-\delta}} I[\mu] \leq I\left[\mu_{a}\right]=\inf _{\mathcal{M}_{a}} I[\mu]$. Therefore one can take $\nu=\mu_{a-\delta}$.

From the strict convexity of $I$ it follows that

$$
I[\nu]>I\left[\mu_{a}\right]+\left\langle D I\left[\mu_{a}\right], \nu-\mu_{a}\right\rangle
$$

where $D I[\mu]=2 U^{\mu}+Q$ is the Fréchet derivative of $I[\mu]$. Therefore, from the properties of $\nu$ we infer

$$
0 \geq I[\nu]-I\left[\mu_{a}\right]>\left\langle D I\left[\mu_{a}\right], \nu-\mu_{a}\right\rangle
$$

or equivalently

$$
\left\langle 2 U^{\mu_{a}}+Q, \nu-\mu_{a}\right\rangle<0
$$

On the other hand

$$
\int\left(2 U^{\mu_{a}}+Q\right) d \mu_{a}=a A_{\Gamma}+(1-a) A_{0}
$$

while

$$
\int\left(2 U^{\mu_{a}}+Q\right) d \nu=\int_{\Gamma}\left(2 U^{\mu_{a}}+Q\right) d \nu+\int_{\mathbb{R}^{2} \backslash \Gamma}\left(2 U^{\mu_{a}}+Q\right) d \nu \geq \nu(\Gamma) A_{\Gamma}+\nu\left(\mathbb{R}^{2} \backslash \Gamma\right) A_{0} .
$$

This together with (3.8), (3.7) yields

$$
a A_{\Gamma}+(1-a) A_{0}>\nu(\Gamma) A_{\Gamma}+(1-\nu(\Gamma)) A_{0} \Rightarrow A_{0}(\nu(\Gamma)-a)>A_{\Gamma}(\nu(\Gamma)-a) .
$$

Finally, the property $\nu(\Gamma)<a$ implies that $A_{\Gamma}>A_{0}$.

Corollary 3.3. $\operatorname{supp} \mu_{a}$ is compact.

Proof. If $d \geq 3$ then $K(x-y) \geq 0$, hence by Lemma 3.2 for $x \in \operatorname{supp} \mu_{a}$ we have

$$
\max \left(A_{\Gamma}, A_{0}\right) \geq 2 U^{\mu_{a}}(x)+Q(x) \geq Q(x) \rightarrow \infty \quad \text { if } \quad|x| \rightarrow \infty
$$

which is a contradiction. If $d=2$ then from the triangle inequality we get that

$$
K(x-y) \geq-\log |x|-\log \left(1+\frac{|y|}{|x|}\right) .
$$

Consequently, for $x \in \operatorname{supp} \mu_{a}$

$$
\begin{aligned}
\max \left(A_{\Gamma}, A_{0}\right) & \geq 2 U^{\mu_{a}}(x)+Q(x) \geq Q(x)-2 \log |x|-\int \log \left(1+\frac{|y|}{|x|}\right) d \mu_{a} \\
& =Q(x)-2 \log |x|+O(1) \rightarrow \infty \quad \text { if } \quad|x| \rightarrow \infty
\end{aligned}
$$


for sufficiently large $|x|$, where the last inequality follows from (4.12) and $\int Q d \mu_{a}<I\left[\mu_{a}\right]<\infty$. Since $Q=|x|^{2}$ (of for the general case from the hypotheses on $Q(\mathbf{H 1})-(\mathbf{H} \mathbf{3})$ ) it again follows that $\operatorname{supp} \mu_{a}$ is bounded.

\section{Global $L^{2}$ estimates for $U^{\mu_{a}}$ And $\nabla U^{\mu_{a}}$}

Our main result is contained in the following

Theorem 4.1. Let $U^{\mu_{a}}(y)=\int K(x-y) d \mu_{a}$, if $d \geq 3$ then $\nabla U^{\mu_{a}} \in L^{2}\left(\mathbb{R}^{d}\right)$. If $d=2$ then $U^{\mu_{a}} \in H^{1}\left(\mathbb{R}^{2}\right)$. Furthermore, there holds

$$
\left\|U^{\mu_{a}}\right\|_{H^{1}\left(\mathbb{R}^{2}\right)} \leq C \mathcal{E}\left[\mu_{a}\right] .
$$

Here $\mathcal{E}[\mu]$ is the energy of $\mu$ defined as $\iint K(x-y) d \mu(x) d \mu(y)$.

Remark 4.2. It is shown in $[3]$ that $\mathcal{E}[\mu]>0$ for any probability measure $\mu$ and $d \geq 2$. In fact, this can be seen from the proof to follow (see also Corollary 4.3).

Proof. The case $d \geq 3$ follows from Lemma 1.6 p. 92 [7] (see also Lemma 17 p. 95), which assert that

$$
\frac{\partial U^{\mu_{a}}(x)}{\partial x_{i}}=\int \frac{\partial K(x-y)}{\partial x_{i}} d \mu_{a}
$$

almost everywhere and morover

$$
\frac{1}{4 \pi^{2}} \int_{\mathbb{R}^{d}}\left|\nabla U^{\mu_{a}}\right|^{2} \leq \iint K(x-y) d \mu_{a}(x) d \mu_{a}(y)=\mathcal{E}\left[\mu_{a}\right] .
$$

The case of the logarithmic potential follows from a modification of the argument by L. Carleson [3] Lemma 3 page 22. We begin with computing the Fourier transformation of $K$. Note that since supp $\mu_{a}$ is compact we can assume that $K(r)=0$ for $r \geq r_{0}$ for some fixed $r_{0}>0$. We have

$$
\begin{aligned}
\widehat{K}(\xi) & =\int K(x) e^{-2 \pi i\langle x, \xi\rangle} d x=\int K(x) e^{-2 \pi i\left\langle x|\xi|, \frac{\xi}{|\xi\rangle}\right.} d x \\
& =\frac{1}{4 \pi^{2}|\xi|^{2}} \int K\left(\frac{y}{2 \pi|\xi|}\right) e^{i\left\langle y, \frac{\xi}{|\xi|}\right\rangle} d x .
\end{aligned}
$$

Let us denote $K_{0}(y)=K\left(\frac{y}{2 \pi|\xi|}\right)$ and define

$$
F(\eta)=\int K_{0}(y) e^{i \pi\langle y, \eta\rangle}, \quad \eta=\frac{\xi}{|\xi|} .
$$

From Lemma 2 p. 21 [3] it follows that there is a universal constant $c_{1}$ such that

$$
F(\eta)=c_{1} \int_{0}^{\infty} K_{0}(r) J(r) r d r, \quad|\eta|=1
$$

where $J$ is the Bessel function

$$
J(r)=-J^{\prime \prime}(r)-\frac{J^{\prime}(r)}{r}, \quad J(0)=1, J^{\prime}(0)=0, \quad J(r)<1, r \neq 0 .
$$


Therefore $F(\eta)$ can be further simplified as follows

$$
\begin{aligned}
F(\eta) & =-c_{1} \int_{0}^{\infty} K_{0}(r)(r J(r))^{\prime} d r= \\
& =c_{1} \int_{0}^{2 \pi|\xi| r_{0}} r J^{\prime}(r) K_{0}^{\prime}(r) d r
\end{aligned}
$$

because from the definition of $K_{0}$ we have supp $K_{0} \subset\left[0,2 \pi|\xi| r_{0}\right]$. Moreover, $K_{0}^{\prime}(r)=-\frac{1}{r}$ hence

$$
F(\eta)=c_{1}\left(1-J\left(2 \pi|\xi| r_{0}\right)\right) .
$$

Consequently,

$$
\widehat{K}(\xi)=\frac{c_{1}}{4 \pi^{2}|\xi|^{2}}\left(1-J\left(2 \pi|\xi| r_{0}\right)\right) .
$$

Next we restrict $\mu_{1}=\mu_{a}\left\llcorner\mathcal{C}\right.$ where $\mathcal{C} \subset \operatorname{supp} \mu_{a}$ is a compact such that $U^{\mu_{1}}$ is continuous. Observe that $\int U^{\mu_{a}} d \mu_{a}$ is finite hence $U^{\mu_{a}}$ is finite $\mu_{a}$ almost everywhere. By Theorem 1.8 p. 70 [7] for every $\varepsilon>0$ small there is a restriction of $\mu_{a}$ such that

$$
0 \leq \int \mu_{a}-\int \mu_{1}<\varepsilon
$$

Note that if $\tau=\mu_{a}-\mu_{1}$ then we have

$$
\left|\mathcal{E}\left[\mu_{a}\right]-\mathcal{E}\left[\mu_{1}\right]\right|=\left|\int U^{\mu_{a}-\mu_{1}} d \mu_{a}+\int U^{\mu_{a}-\mu_{1}} d \mu_{1}\right|=\left|\int\left(U^{\mu_{a}}+U^{\mu_{1}}\right) d \tau\right|=O(\varepsilon) .
$$

Let $\phi_{n}(y)=n^{\frac{d}{2}} e^{-n \pi|y|^{2}}$ be the sequence of normalised Gaussian kernels. It is well-known that $\phi_{n}$ is a mollification kernel for every $n \in \mathbb{N}$ and moreover $\widehat{\phi_{n}}=e^{-\frac{\phi|\xi|^{2}}{n}}$. From the Parseval relation

$$
\int\left(\phi_{n} * U^{\mu_{1}}\right) d \mu_{1}=\int \widehat{\phi_{n}} \widehat{K}\left|\widehat{\mu_{1}}\right|^{2} .
$$

If we first send $n \rightarrow \infty$ and then $\varepsilon \rightarrow 0$ to conclude the identity

$$
\mathcal{E}\left[\mu_{a}\right]=\int \widehat{K}\left|\widehat{\mu_{a}}\right|^{2} .
$$

On the other hand $\widehat{U^{\mu_{a}}}=\widehat{K} \widehat{\mu_{a}}$, which yields

$$
\begin{aligned}
\mathcal{E}\left[\mu_{a}\right] & =\int \widehat{K}(\xi) \frac{\left|\widehat{U \mu_{a}}(\xi)\right|^{2}}{|\widehat{K}(\xi)|^{2}} d \xi \\
& =\int \frac{4 \pi^{2}|\xi|^{2}}{c_{1}\left(1-J\left(2 \pi r_{0}|\xi|\right)\right)}\left|\widehat{U \mu_{a}}(\xi)\right|^{2} d \xi \\
& =\int_{|\xi|<\delta}+\int_{|\xi| \geq \delta} .
\end{aligned}
$$

Using the expansion $J(t)=\sum_{s=0}^{\infty} \frac{(-1)^{s}}{(s !)^{2}}\left(\frac{t}{2}\right)^{2 s}=1-\frac{t^{2}}{4}+\frac{t^{4}}{64}+\ldots$ we see that

$$
\frac{4 \pi^{2}|\xi|^{2}}{c_{1}\left(1-J\left(2 \pi r_{0}|\xi|\right)\right)}=\frac{1}{r_{0}^{2} c_{1}} \frac{4}{\left(1-\frac{\left(2 \pi r_{0}|\xi|\right)^{2}}{16}+\ldots\right)}
$$


hence the first integral is bounded below by $C(\delta) \frac{1}{r_{0}^{2} c_{1}} \int_{|\xi|<\delta}\left|\widehat{U^{\mu_{a}}}(\xi)\right|^{2} d \xi$ for sufficiently small $\delta>0$. As for the second integral, we have

$$
\int_{|\xi| \geq \delta} \frac{4 \pi^{2}|\xi|^{2}}{c_{1}\left(1-J\left(2 \pi r_{0}|\xi|\right)\right)}\left|\widehat{U^{\mu_{a}}}(\xi)\right|^{2} d \xi \geq \frac{4 \pi^{2} \delta^{2}}{c_{1}} \int_{|\xi| \geq \delta}\left|\widehat{U^{\mu_{a}}}(\xi)\right|^{2} d \xi .
$$

Combining we see that $\widehat{U^{\mu_{a}}} \in L^{2}\left(\mathbb{R}^{2}\right)$ which, after we apply Parseval's relation again, yields $U^{\mu_{a}} \in L^{2}\left(\mathbb{R}^{2}\right)$ and

$$
\left\|U^{\mu_{a}}\right\|_{L^{2}\left(\mathbb{R}^{2}\right)} \leq C \mathcal{E}\left[\mu_{a}\right] .
$$

To finish the proof we use that $4 \pi^{2}|\xi|^{2}\left|\widehat{U^{\mu_{a}}}\right|^{2}=\left|\widehat{\nabla U^{\mu_{a}}}\right|^{2}$ which together with (4.8) implies that

$$
\mathcal{E}\left[\mu_{a}\right]=\int \frac{1}{c_{1}\left(1-J\left(2 \pi r_{0}|\xi|\right)\right)}\left|\widehat{\nabla U^{\mu_{a}}}(\xi)\right|^{2} d \xi \geq \frac{1}{c_{1}} \int\left|\widehat{\nabla U^{\mu_{a}}}(\xi)\right|^{2} d \xi
$$

which finishes the proof.

Corollary 4.3. Let $\mu_{a}$ be as in Theorem 2.1. Then there holds

$$
\mathcal{E}\left[\mu_{a}\right]=\int U^{\mu_{a}} d \mu_{a}>0 .
$$

\section{The THin OBSTACLE PROBlem}

From the $H^{1}\left(\mathbb{R}^{2}\right)$ estimate for $U^{\mu_{a}}$ it follows that $U^{\mu_{a}}$ is a solution to some variational inequality, and hence $U^{\mu_{a}}$ can be interpreted as a solution to an obstacle problem with a combination of both thin (on $\Gamma$ ) and "thick" obstacles (on $\mathbb{R}^{2} \backslash \Gamma$ ). It is convenient to define the obstacle as follows

$$
\psi(x)= \begin{cases}\frac{1}{2}\left(A_{\Gamma}-|x|^{2}\right) & \text { if } x \in \Gamma, \\ \frac{1}{2}\left(A_{0}-|x|^{2}\right) & \text { if } x \in \mathbb{R}^{2} \backslash \Gamma .\end{cases}
$$

Lemma 5.1. Let $U^{\mu_{a}}$ be the logarithmic potential of $\mu_{a}$ and define

$$
\mathcal{K}=\left\{v \in H_{\text {loc }}^{1}\left(\mathbb{R}^{2}\right) \text { s.t. } v-U^{\mu_{a}} \text { has bounded support in } \mathbb{R}^{2}, v \geq \psi\right\} .
$$

Then $U^{\mu_{a}}$ solves the following obstacle problem:

$$
\int \nabla U^{\mu_{a}} \nabla\left(v-U^{\mu_{a}}\right) \geq 0, \quad \forall v \in \mathcal{K} .
$$

The proof is the same as in [2].

Corollary 5.2. $\operatorname{dist}\left(\Gamma, \operatorname{supp}\left(\mu_{a} \backslash \Gamma\right)\right)>0$.

Proof. This follows from the estimate $A_{\Gamma}>A_{0}$. Indeed, let us assume that $x_{0} \in \Gamma \cap \operatorname{supp} \mu_{a}$ and there is a sequence $\left\{x_{k}\right\}_{k=1}^{\infty}, x_{k} \in \operatorname{supp} \mu_{a} \backslash \Gamma$ such that $\lim _{k \rightarrow \infty} x_{k} \rightarrow x_{0}$. Using the lower semicontinuity of $U^{\mu_{a}}$ (see Lemma 1 p.15 [3]) we see that

$$
\frac{1}{2}\left(A_{0}-\left|x_{0}\right|^{2}\right)=\liminf _{x_{k} \rightarrow x_{0}} U^{\mu_{a}}\left(x_{k}\right) \geq U^{\mu_{a}}\left(x_{0}\right) .
$$

Let $\rho>0$ be such that $\left\{x_{k}\right\} \subset B_{\rho}\left(x_{0}\right)$. If $\rho$ is small then $\Gamma$ divides $B_{\rho}\left(x_{0}\right)$ into two parts $D^{+}$and $D^{-}$. To fix the ideas let us suppose that $D^{+}$contains a subsequence $\left\{x_{k}\right\}$. Let $h$ be the harmonic function in $D^{+}$ 
such that $h=\psi$ on $\partial D^{+}$. Observe that $h$ is continuous at $x_{0}$ because $\Gamma \in C^{1, \alpha}$. Since $U^{\mu_{a}}$ is superharmonic and on $\partial D^{+}$we have $U^{\mu_{a}} \geq \psi=h$ then the comparison principle implies that

$$
U^{\mu_{a}}\left(x_{0}\right) \geq h\left(x_{0}\right)=\frac{1}{2}\left(A_{\Gamma}-\left|x_{0}\right|^{2}\right) .
$$

Combining (5.2) and (5.3) we see that $A_{0} \geq A_{\Gamma}$ which is a contradiction in view of (3.5).

From Corollary 5.2 it follows that near $\Gamma$ the potential $U^{\mu_{a}}$ is a solution to a thin obstacle problem in the following sense, see [5] p. 108:

$$
\left.\begin{array}{l}
U^{\mu_{a}} \geq \frac{1}{2}\left(A_{\Gamma}-Q\right) \\
\frac{\partial U^{\mu_{a}}}{\partial n^{+}}+\frac{\partial U^{\mu_{a}}}{\partial n^{-}} \geq 0 \\
\left(u-\frac{1}{2}\left(A_{\Gamma}-Q\right)\right)\left(\frac{\partial U^{\mu_{a}}}{\partial n^{+}}+\frac{\partial U^{\mu_{a}}}{\partial n^{-}}\right)=0
\end{array}\right\} \text { on } \Gamma
$$

where $n^{ \pm}$are the outward normals on the $\Gamma$ corresponding to the domains that $\Gamma$ separates. In particular, if $\Gamma$ is $C^{3}$ regular then $U^{\mu_{a}}$ is $C^{1, \alpha}$ up to $\Gamma$ from each of its side, see Theorem 11.4 p.111 [5].

A particular case is $\Gamma=\mathbb{R}$ [4]. Using a simple symmetrization argument (see e.g. [6] p. 119 Theorem 4.6) we can show that the potential $U^{\mu_{a}}$ is symmetric w.r.t. the real line and hence we get the Signorini problem near $\mathbb{R}[5]$ p. 111 .

One can make the connections with the obstacle problem more explicit by using the $H^{1}\left(\mathbb{R}^{2}\right)$ estimate in Theorem 4.1 and transforming the energy $I\left[\mu_{a}\right]$. Let $R>0$ be fixed then using the divergence theorem

$$
\begin{aligned}
\int_{B_{R}} U^{\mu_{a}} d \mu_{a} & =-\frac{1}{2 \pi} \int_{B_{R}} U^{\mu_{a}} \Delta U^{\mu_{a}}= \\
& =\frac{1}{2 \pi} \int_{B_{R}}\left|\nabla U^{\mu_{a}}\right|^{2}-\frac{1}{2 \pi} \int_{\partial B_{R}} U^{\mu_{a}} \partial_{n} U^{\mu_{a}}
\end{aligned}
$$

For a.e. $R>0$ the last integral can be estimated as follows

$$
\left|\int_{\partial B_{R}} U^{\mu_{a}} \partial_{n} U^{\mu_{a}}\right| \leq \int_{\partial B_{R}}\left|U^{\mu_{a}}\right|\left|\nabla U^{\mu_{a}}\right| \leq \int_{\partial B_{R}}\left|U^{\mu_{a}}\right|^{2}+\left|\nabla U^{\mu_{a}}\right|^{2} .
$$

From Theorem 4.1 and Fubini's theorem it follows that

$$
\int_{R^{2}}\left(\left|U^{\mu_{a}}\right|^{2}+\left|\nabla U^{\mu_{a}}\right|^{2}\right)=\int_{0}^{\infty} \int_{\partial B_{R}}\left(\left|U^{\mu_{a}}\right|^{2}+\left|\nabla U^{\mu_{a}}\right|^{2}\right) d R .
$$

Consequently,

and we infer from (5.5) that

$$
\int_{\partial B_{R}}\left|U^{\mu_{a}}\right|^{2}+\left|\nabla U^{\mu_{a}}\right|^{2} \rightarrow 0 \quad R \rightarrow \infty
$$

$$
\int_{\mathbb{R}^{2}} U^{\mu_{a}} d \mu_{a}=\frac{1}{2 \pi} \int_{\mathbb{R}^{2}}\left|\nabla U^{\mu_{a}}\right|^{2}
$$

Recalling that by Corollary $3.3 \operatorname{supp} \mu_{a} \subset B_{r_{0}}$ for some $r_{0}>0$ and using the divergence theorem again we conclude

$$
\begin{aligned}
\int_{B_{r_{0}}}|x|^{2} d \mu_{a} & =-\frac{1}{2 \pi} \int_{B_{r_{0}}}|x|^{2} \Delta U^{\mu_{a}}=-\frac{1}{2 \pi} \int_{B_{r_{0}}} U^{\mu_{a}} \Delta|x|^{2}+\frac{1}{2 \pi} \int_{\partial B_{r_{0}}}\left(2 r_{0} U^{\mu_{a}}-r_{0}^{2} \partial_{n} U^{\mu_{a}}\right) \\
& =-\frac{2}{\pi} \int_{B_{r_{0}}} U^{\mu_{a}}+\frac{r_{0}}{\pi} \int_{\partial B_{r_{0}}} U^{\mu_{a}}+r_{0}^{2}
\end{aligned}
$$


Combining these we have that the energy can be rewritten in terms of $U^{\mu_{a}}$ in the following form

$$
I\left[\mu_{a}\right]=\frac{1}{2 \pi} \int_{\mathbb{R}^{2}}\left|\nabla U^{\mu_{a}}\right|^{2}-\frac{2}{\pi} \int_{B_{r_{0}}} U^{\mu_{a}}+\frac{r_{0}}{\pi} \int_{\partial B_{r_{0}}} U^{\mu_{a}}+r_{0}^{2} .
$$

\section{REFERENCES}

[1] H.W. Alt, L. A. Caffarelli: Existence and regularity for a minimum problem with free boundary, Journal für die Reine und Ange. Math. 325 (1981), 105-144.

[2] S. Armstrong, S. Serfaty, O. Zeitouni: Remarks on a constrained optimization problem for the Ginibre ensemble. Potential Anal. 41 (2014), no. 3, 945-958

[3] L. Carleson: Selected problems on exceptional sets. Van Nostrand Mathematical Studies, No. 13 D. Van Nostrand Co., Inc., Princeton, N.J.-Toronto, Ont.-London, 1967

[4] L.C.G. Del Molino, K. Pakdaman, J. Touboul, G. Wainrib: The real Ginibre ensemble with $k=O(n)$ real eigenvalues, Journal of Statistical Physics April 2016, Volume 163, Issue 2, pp 303-323

[5] A. Friedman: Variational Principles and Free-Boundary Problems (Dover Books on Mathematics), 2010

[6] W. Hayman: Multivalent functions, Cambridge University Press, 1993.

[7] N. Landkof: Foundations of modern potential theory. Translated from the Russian by A. P. Doohovskoy. Die Grundlehren der mathematischen Wissenschaften, Band 180. Springer-Verlag, New York-Heidelberg, 1972

[8] E. SAFF, V. TотіK: Logarithmic potentials with external fields, Springer, 1997.

School of Mathematics, The University of Edinburgh, Peter Tait Guthrie Road, EH9 3FD, Edinburgh, UK

E-mail address: aram6k@gmail.com 